NISSUNA UMANA INVESTIGAZIONE SI PUO DIMANDARE VERA SCIENZIA S'ESSA NON PASSA PER LE MATEMATICHE DIMOSTRAZIONI LEONARDO DA VINCI

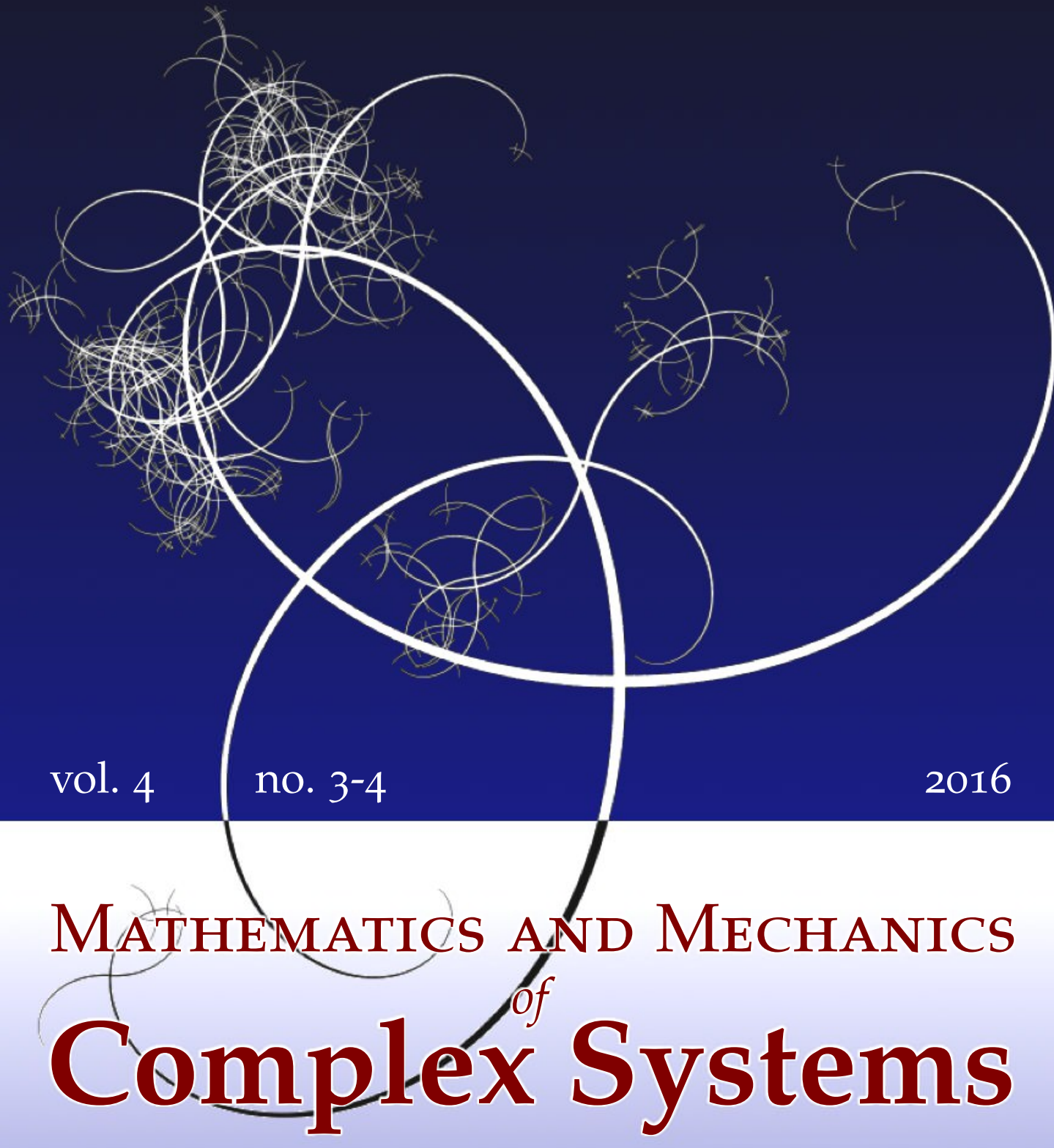

Mario Pulvirenti ANd SERgio Simonella PROPAGATION OF CHAOS AND EFFECTIVE EQUATIONS IN KINETIC THEORY: A BRIEF SURVEY 


\title{
PROPAGATION OF CHAOS AND EFFECTIVE EQUATIONS IN KINETIC THEORY: A BRIEF SURVEY
}

\author{
Mario Pulvirenti And SERgio Simonella
}

\begin{abstract}
We review some historical highlights leading to the modern perspective on the concept of chaos from the point of view of kinetic theory. We focus in particular on the role played by the propagation of chaos in the mathematical derivation of effective equations.
\end{abstract}

\section{The paradigm of kinetic theory}

Propagation of chaos is a central topic in kinetic theory and certainly exhibits interesting features from the point of view of probability theory and mathematical physics.

This contribution is dedicated to our friend and colleague Lucio Russo, who gave and is giving important contributions to these fields and to the history of science. The purpose is to review some important steps in the mathematical understanding of kinetic equations and of the notion of chaos.

We do not pretend to be exhaustive and limit ourselves to a selection of arguments which played a key role from a modern outlook. We also comment on some perhaps less known historical aspects underlining the long and difficult path of the scientific progress.

Many interesting systems in physics and applied sciences consist of a large number of identical components so that they are difficult to analyze from a mathematical point of view. On the other hand, quite often, we are not interested in a detailed description of the system but rather in its collective behavior. Therefore, it is necessary to look for all procedures leading to simplified models, retaining the interesting features of the original system, cutting away redundant information. This is exactly the methodology of statistical mechanics and kinetic theory. Here we want to outline the limiting procedure leading from the microscopic description of a large particle system (based on the fundamental laws like the Newton or Schrödinger equations) to the more practical picture dictated by kinetic theory.

\section{Communicated by Raffaele Esposito.}

MSC2010: 82-03.

Keywords: propagation of chaos, Boltzmann, Landau and Vlasov equations. 
Although recently the methodology of kinetic theory has been applied to a large variety of complex systems (consisting of a huge number of individuals), we will discuss here only models arising in physics and more precisely in classical mechanics. The starting point is a system of $N$ identical particles in the space $\mathbb{R}^{3}$. A microscopic state of the system is a sequence $z_{1}, \ldots, z_{N}$ where $z_{i}=\left(x_{i}, v_{i}\right)$ denotes position and velocity of the $i$-th particle. The particles interact via the (smooth) two-body interaction $\varphi: \mathbb{R}^{3} \rightarrow \mathbb{R}$, and the equations of motion are

$$
\left\{\begin{array}{l}
\dot{x}_{i}=v_{i} \\
\dot{v}_{i}=-\sum_{j: j \neq i} \nabla \varphi\left(x_{i}-x_{j}\right)
\end{array}\right.
$$

Particles have unit mass, and $\varphi$ depends on the distance $\left|x_{i}-x_{j}\right|$ so that the force of particle $j$ acting on particle $i$ (that is, $-\nabla \varphi\left(x_{i}-x_{j}\right)$ ) is directed along $x_{i}-x_{j}$.

We are interested in a situation where $N$ is very large (for instance, a cubic centimeter of a rarefied gas contains approximately $10^{19}$ molecules). The knowledge of the microscopic states becomes useless, and we turn to a statistical description. We introduce a probability measure $W^{N}\left(Z_{N}\right) d Z_{N}$ (absolutely continuous with respect to the Lebesgue measure), defined on the phase space of the system $\mathbb{R}^{3 N} \times \mathbb{R}^{3 N}$, where

$$
Z_{N}=\left(z_{1}, \ldots, z_{N}\right)=\left(x_{i}, v_{i}, \ldots, x_{N}, v_{N}\right)
$$

$W^{N}$ assigns the same statistical weight to two different vectors $Z_{N}$ and $Z_{N}^{\prime}$ differing only for the order of particles, i.e., identifying the same physical configuration. Physically relevant measures are symmetric with respect to permutations of the sequence $z_{1}, \ldots, z_{N}$.

The time-evolved measure is defined by

$$
W^{N}\left(Z_{N}, t\right)=W^{N}\left(\Phi^{-t}\left(Z_{N}\right)\right) .
$$

Here $\Phi^{t}\left(Z_{N}\right)$ denotes the dynamical flow constructed by solving the equations of motion; namely, $\Phi^{t}\left(Z_{N}\right)$ solves (1) with initial datum $Z_{N}$.

We can establish a partial differential equation, called the Liouville equation, describing the evolution of the measure (2). However, this equation is also not tractable from a practical point of view. To have an efficient reduced description, one can focus on the time evolution for the probability distribution of a given particle (say particle 1), all the particles being identical.

To this end, we define the $j$-particle marginals

$$
f_{j}^{N}\left(Z_{j}, t\right)=\int d z_{j+1} \cdots d z_{N} W^{N}\left(Z_{j}, z_{j+1}, \ldots, z_{N}, t\right), \quad j=1, \ldots, N,
$$


and we look for an equation describing the evolution of $f_{1}^{N}$. Roughly, we establish an evolution equation of the form

$$
\partial_{t} f_{1}^{N}=-v \cdot \nabla f_{1}^{N}+Q .
$$

The first term in the right-hand side denotes the contribution to the evolution of $f_{1}^{N}$ due to the free transport of particles, while the term $Q$ should describe the interaction of particle 1 with the rest of the system.

We now face a big difficulty. Since the interaction is binary, $Q$ will depend on $f_{2}^{N}$, namely the two-particle marginal. In other words, (4) is still useless because to know $f_{1}^{N}$ we need to know $f_{2}^{N}$ and to know $f_{2}^{N}$ we need to know $f_{3}^{N}$ and so on. We handle a hierarchy of equations, called BBGKY hierarchy [Bogolyubov 1946] (from the names of the physicists Bogolyubov, Born, Green, Kirkwood, and Yvon).

Here enters the property called propagation of chaos, that is,

$$
f_{2}^{N}\left(x_{1}, v_{1}, x_{2}, v_{2}, t\right)=f_{1}^{N}\left(x_{1}, v_{1}, t\right) f_{1}^{N}\left(x_{2}, v_{2}, t\right) .
$$

Accepting (5), $Q$ becomes a bilinear operator of $f_{1}^{N}$ and (4) is a closed equation. We have thus replaced a huge ordinary differential system with a single PDE. The price we pay is that (4) is nonlinear.

Strictly speaking, (5) is certainly false since it expresses the statistical independence of particle 1 and particle 2, which even if assumed at time 0 cannot hold at later times. Indeed, the dynamics creates correlations. Nevertheless, one can hope to recover this property in some asymptotic situation described by a suitable scaling limit. This is what happens in three different physical contexts: the meanfield, the low-density and the weak-coupling limits, yielding three different kinetic equations, namely the Vlasov, Boltzmann and Landau equations, respectively.

\section{Mean-field limit and Vlasov equation}

The simplest example in which the methods of kinetic theory apply is the meanfield limit. Let us suppose that the particle system we are considering interacts through a very small (possibly long-range) potential $O(1 / N)$, where the number of particles $N$ is going to diverge. The equation of motion becomes

$$
\left\{\begin{array}{l}
\dot{x}_{i}=v_{i}, \\
\dot{v}_{i}=-(1 / N) \sum_{j: j \neq i} \nabla \varphi\left(x_{i}-x_{j}\right) .
\end{array}\right.
$$

Consider also an initial distribution $W^{N}$ fully factorized, i.e., $W^{N}=f_{0}^{\otimes N}$. In this situation, the dynamics creates correlations at each positive time. However, given two particles, say 1 and 2 , the dynamics of particle 1 is influenced by the presence of particle 2 by a factor $O(1 / N)$. The same happens for particle 2 regarding the influence of particle 1 . Therefore, we expect that the correlations are negligible 
and in the limit $N \rightarrow \infty$

$$
f_{2}^{N} \approx\left(f_{1}^{N}\right)^{\otimes 2}
$$

We shall see that, in our context, (4) becomes

$$
\left(\partial_{t}+v_{1} \cdot \nabla_{x_{1}}\right) f_{1}^{N}(t)=\frac{(N-1)}{N} \int d x_{2} \int d v_{2} \nabla \varphi\left(x_{1}-x_{2}\right) \cdot \nabla_{v_{1}} f_{2}^{N}\left(x_{1}, v_{1}, x_{2}, v_{2}, t\right)
$$

so that, using (7) and formally taking the limit $N \rightarrow \infty$, we arrive at the following equation for the one-particle distribution $f$ :

$$
\left(\partial_{t}+v \cdot \nabla_{x}\right) f(x, v, t)=\int d y \int d w f(y, w, t) \nabla \varphi(x-y) \cdot \nabla_{v} f(x, v, t) .
$$

Equation (9) is called the Vlasov equation (from the name of the physicist who introduced it) and describes a large system of weakly interacting particles.

The rigorous analysis of the mean-field limit and the Vlasov equation are a well understood subject in the case of smooth potentials (see for instance [Dobrušin 1979]). The interesting case of the Coulomb interaction is still a challenging open problem.

\section{The Boltzmann equation}

Much more subtle are the limiting physical situations leading to the Boltzmann equation.

Ludwig Boltzmann established an evolution equation to describe the behavior of a rarefied gas in 1872, starting from the mathematical model of elastic balls and using mechanical and statistical considerations. The importance of this equation is twofold. On one side, it provides (as well as the hydrodynamical equations) a reduced description of the microscopic world. On the other, it is also an important tool for applications, especially for dilute fluids when the hydrodynamical equations fail to hold.

According to the general paradigm of kinetic theory, the starting point of Boltzmann's analysis is to renounce the study of gas in terms of the detailed motion of the molecules of the full system. It is preferable to investigate a function $f(x, v)$ which is the probability density of a given particle, where $x$ and $v$ denote its position and velocity.

Following the original approach proposed by Boltzmann, $f(x, v) d x d v$ is to rather be interpreted as the fraction of molecules falling in the cell of the phase space of size $d x d v$ around $(x, v)$. The two concepts are not exactly the same, but they are asymptotically equivalent (when the number of particles diverges) if a law of large numbers holds. 
More precisely, Boltzmann considered the occupation numbers of the cells of a grid in the phase space, when the side of the cell is macroscopically small but sufficiently large to contain a huge number of particles. From a historical point of view, Boltzmann's analysis was really remarkable. Probability theory was not well developed from a mathematical point of view, and even the possibility of describing the macroscopic world in terms of atoms and molecules was still doubtful.

Boltzmann considered a gas as microscopically described by a system of elastic (hard) balls, colliding according to the laws of classical mechanics.

The Boltzmann equation for the one-particle distribution function reads

$$
\left(\partial_{t}+v \cdot \nabla_{x}\right) f=Q(f, f)
$$

where $Q$, the collision operator, is defined by $Q(f, f)(x, v)=\int_{\mathbb{R}^{3}} d v_{1} \int_{S_{+}^{2}} d n\left(v-v_{1}\right) \cdot n\left[f\left(x, v^{\prime}\right) f\left(x, v_{1}^{\prime}\right)-f(x, v) f\left(x, v_{1}\right)\right]$,

with

$$
\begin{aligned}
v^{\prime} & =v-n\left[n \cdot\left(v-v_{1}\right)\right], \\
v_{1}^{\prime} & =v_{1}+n\left[n \cdot\left(v-v_{1}\right)\right]
\end{aligned}
$$

and $n$ a unitary vector varying in $S_{+}^{2}=\left\{n: n \cdot\left(v-v_{1}\right) \geq 0\right\}$.

Note that $v^{\prime}$ and $v_{1}^{\prime}$ are the outgoing velocities after a collision of two elastic balls with incoming velocities $v$ and $v_{1}$ and centers $x$ and $x+\varepsilon n$, with $\varepsilon$ the diameter of the spheres. Clearly the collision takes place if $n \cdot\left(v-v_{1}\right)>0$. Formulas (12) are consequences of the conservation of energy and momentum. Note that $\varepsilon$ does not enter (10) as a parameter.

As a fundamental feature of (10), one has the formal conservation (in time) of the five quantities

$$
\int d x \int d v f(x, v ; t) v^{\alpha}
$$

with $\alpha=0,1,2$, expressing conservation of probability, momentum and energy, respectively. From now on, we shall set $\int=\int_{\mathbb{R}^{3}}$ for notational simplicity.

Moreover, Boltzmann introduced the (kinetic) entropy defined by

$$
H(f)=\int d x \int d v f \log f(x, v)
$$

and proved the famous $H$ theorem asserting the decrease of $H(f(t))$ along the solutions of (10).

Finally, in the case of bounded domains or homogeneous solutions $(f=f(v, t)$ is independent of $x$ ), the distribution defined for some $\beta>0, \rho>0$ and $u \in \mathbb{R}^{3}$ by

$$
M(v)=\frac{\rho}{(2 \pi / \beta)^{3 / 2}} e^{-(\beta / 2)|v-u|^{2}},
$$


called the Maxwellian distribution, is stationary for the evolution given by (10). In addition, $M$ minimizes $H$ among all distributions with given total mass $\rho$, mean velocity $u$ and mean energy. The parameter $\beta$ is interpreted as the inverse temperature.

In conclusion, Boltzmann was able to introduce an evolution equation with the remarkable properties of expressing mass, momentum and energy conservation and also the tendency to thermal equilibrium. In other words, he tried to conciliate Newton's laws with the second principle of thermodynamics.

The $H$ theorem is in contrast with the laws of mechanics, which are timereversible. This fact caused skepticism among the scientific community, and the work of Boltzmann was attacked by several scientists. We refer the reader to the beautiful monograph by C. Cercignani [1998], which is a marvelous compromise between historical and high-level scientific divulgation, to have a faithful idea of the debate at the time.

To formally derive (10), let us consider a system of $N$ identical hard spheres of diameter $\varepsilon$ and unitary mass, interacting by means of the collision law (12). We denote by $\varepsilon$ the diameter of the particles in view of the fact that $\varepsilon$ is very small compared with typical macroscopic lengths.

The phase space $\Gamma_{N}$ of the system is the subset of $\left(\mathbb{R}^{6}\right)^{N}$ satisfying the hard-core condition, namely

$$
\left|x_{i}-x_{j}\right| \geq \varepsilon \quad \text { for } i \neq j .
$$

The dynamical flow is defined as the free flow, i.e.,

$$
Z_{N}(t)=\left(x_{1}+v_{1} t, v_{1}, \ldots, x_{N}+v_{N} t, v_{N}\right)
$$

up to the first impact time, namely when $\left|x_{i}-x_{j}\right|=\varepsilon$. Then an instantaneous collision takes place according to the law (12), and the flow goes on up to the next collision instant.

We denote by $Z_{N} \rightarrow \Phi^{t}\left(Z_{N}\right)$ the dynamical flow constructed in this way. The well-posedness of the hard-sphere dynamics is not obvious, due to the occurrence of multiple collisions or to the a priori possibility that collision times accumulate at a finite limiting time. However, such pathologies cannot occur outside a set of initial conditions $Z_{N}$ of vanishing measure. Therefore, the flow $Z_{N} \rightarrow \Phi^{t}\left(Z_{N}\right)$ can be defined almost everywhere with respect to the Lebesgue measure, and this is enough for our purposes.

Given a probability measure with density $W_{0}^{\varepsilon}$ on $\Gamma_{N}$, thanks to the invariance of the Lebesgue measure under the above evolution, we define the time-evolved measure as the measure with density

$$
W^{\varepsilon}\left(Z_{N}, t\right)=W_{0}^{\varepsilon}\left(\Phi^{-t} Z_{N}\right) .
$$


We recall that we consider probability distributions $W_{0}^{\varepsilon}$ which are initially (and hence at any positive time) symmetric in the exchange of the particles. The probability density of the first $j$ particles is given by the $j$-particle marginal

$$
f_{j}^{\varepsilon}\left(Z_{j}, t\right)=\int d z_{j+1} \cdots d z_{N} W^{\varepsilon}\left(Z_{j}, z_{j+1}, \ldots, z_{N}, t\right), \quad j=1, \ldots, N .
$$

Notational remark. Up to now, the two parameters $N$ and $\varepsilon$ have been introduced independently, and the definitions $\Gamma_{N}, Z_{N}, W^{\varepsilon}, f_{j}^{\varepsilon}$, etc., should exhibit a double dependence. However, in a moment, we shall fix a precise dependence $\varepsilon=\varepsilon(N)$ so that the notation is unambiguous.

Cercignani [1972] derived a hierarchy of equations for the marginals, and the first of such equations, for the one-particle distribution, is

$$
\left(\partial_{t}+v \cdot \nabla_{x}\right) f_{1}^{\varepsilon}=\text { Coll, }
$$

where Coll denotes the variation of $f$ due to the collisions. It takes the form

$$
\text { Coll }=(N-1) \varepsilon^{2} \int d v_{2} \int_{S^{2}} d n f_{2}^{\varepsilon}\left(x, v, x+n \varepsilon, v_{2}\right)\left(v_{2}-v\right) \cdot n .
$$

Let us argue the physical significance of (19) and (18). In absence of collisions, the probability density of a given particle would be conserved, that is,

$$
\frac{d}{d t} f^{\varepsilon}(x+v t, v, t)=0 .
$$

The presence of collisions and the total conservation of the probability imply that

$$
\frac{d}{d t} f^{\varepsilon}(x+v t, v, t)=\text { flux }
$$

where the flux is computed on the boundary of the spherical surface of the ball of radius $\varepsilon$ around $x$. Therefore, the probability flux due to a collision with a given particle, say particle 2 , having velocity $v_{2}$, is given by

$$
-\varepsilon^{2} \int_{S^{2}} f_{2}^{\varepsilon}\left(x, v, x+n \varepsilon, v_{2}\right) V \cdot n
$$

where $V=v_{2}-v$ is the relative velocity and $-n$ is the inward normal to the considered surface.

Integrating with respect to $d v_{2}$ and summing over all the possible choices of particles, we arrive to (18) and (19).

This is, basically, Boltzmann's original argument, except for an important conceptual difference. The basic object of investigation considered by Boltzmann is not the probability density $f$ but rather the quantity

$$
f^{\varepsilon}(x, v, t) \approx \frac{N_{\Delta}(t)}{|\Delta| N}
$$


where $\Delta$ is a small cell on the phase space around the point $(x, v),|\Delta|$ is its volume and $N_{\Delta}(t)$ is the occupation number of the cell $\Delta$ (number of particles falling in $\Delta$ ) at time $t$. Clearly, $\Delta$ must be small compared with the macroscopic lengths, e.g., the size of the box in which the gas is confined, but large with respect to the typical microscopic lengths, for instance $\varepsilon$ (molecular diameter).

The two concepts introduced are not exactly the same, but in view of a limit $N \rightarrow \infty$, it is possible to conceive a law of large numbers allowing one to identify the empirical sample with the a priori probability.

Next we shall tackle the problem of getting a closed equation. Apparently, we are in a situation analogous to the one discussed for the Vlasov equation, but there is a deep difference. Indeed for the hard-sphere system, one can write a hierarchy of equations which plays the role of the BBGKY hierarchy for smooth potentials. However, the interaction among the particles is strong and the mean-field argument used to invoke the propagation of chaos fails.

Boltzmann's most important assumption enters here, namely that two given particles should be (almost) uncorrelated if the gas is rarefied enough. This leads to the propagation of chaos

$$
f_{2}^{\varepsilon}\left(x, v, x_{2}, v_{2}\right)=f^{\varepsilon}(x, v) f^{\varepsilon}\left(x_{2}, v_{2}\right),
$$

which is however much more delicate in the present context. In fact, if two particles collide, correlations are created. Even assuming (20) at some time, if particle 1 collides with particle 2 , such an equation cannot be satisfied at any time after the collision.

Before discussing the propagation of chaos further, we notice that, in practical situations, for a rarefied gas, the combination $N \varepsilon^{3} \approx 10^{-8} \mathrm{~cm}^{3}$ (total volume occupied by the particles) is very small, while $N \varepsilon^{2}=O(1)$. This implies that the collision operator given by (19) is $O(1)$. Therefore, since we are dealing with a huge number of particles, we are tempted to perform the limit $N \rightarrow \infty$ and $\varepsilon \rightarrow 0$ in such a way that $\varepsilon^{2}=O\left(N^{-1}\right)$. As a consequence, the probability that two tagged particles collide (which is of the order of the surface of a ball, that is, $O\left(\varepsilon^{2}\right)$ ) is negligible. Instead, the probability that a given particle collides with any of the remaining $N-1$ particles $\left(O\left(N \varepsilon^{2}\right)=O(1)\right)$ is not negligible. On the other hand, condition (20) refers to two preselected particles (say 1 and 2) and it is not unreasonable to conceive that it holds in the limiting situation in which we are working.

Nevertheless, we cannot insert (20) into (19) because the integral operator refers to times both before and after the collision. Let us assume (20) only when the pair of velocities $v$ and $v_{2}$ are incoming $\left(\left(v-v_{2}\right) \cdot n>0\right)$. If the two particles are initially uncorrelated, it is unlikely that they have collided before a given time $t$ so that we assume their statistical independence. 
This is a standard argument in textbooks of kinetic theory, but some extra comments are needed. If particles 1 and 2 have not collided directly before a given time $t$, this does not imply that they are uncorrelated. Indeed there may exist a chain of collisions involving a group $i_{1}, i_{2}, \ldots$ of particles

$$
1 \rightarrow i_{1} \rightarrow i_{2} \rightarrow \cdots \rightarrow 2
$$

correlating particles 1 and 2 . The occurrence of this event must be excluded by a rigorous mathematical analysis.

Coming back to (19), for the outgoing pair of velocities $v$ and $v_{2}\left(\left(v_{2}-v\right) \cdot n>0\right)$, we make use of the continuity property

$$
f_{2}^{\varepsilon}\left(x, v, x+n \varepsilon, v_{2}\right)=f_{2}^{\varepsilon}\left(x, v^{\prime}, x+n \varepsilon, v_{2}^{\prime}\right)
$$

where the pair $v^{\prime}$ and $v_{2}^{\prime}$ is precollisional. On the two-particle distribution expressed in terms of precollisional variables, we apply condition (20), obtaining

$$
\begin{aligned}
\text { Coll }=(N-1) \varepsilon^{2} \int d v_{2} & \int_{S_{+}^{2}} d n\left(v-v_{2}\right) \cdot n \\
& \times\left[f\left(x, v^{\prime}\right) f\left(x-n \varepsilon, v_{2}^{\prime}\right)-f(x, v) f\left(x+n \varepsilon, v_{2}\right)\right]
\end{aligned}
$$

after a change $n \rightarrow-n$ in the positive part of Coll, using the notation $S_{+}^{2}$ for the hemisphere $S_{+}^{2}=\left\{n: n \cdot\left(v-v_{2}\right) \geq 0\right\}$.

Finally, in the limit as $N \rightarrow \infty$ and $\varepsilon \rightarrow 0$ with $N \varepsilon^{2}=\lambda^{-1}$, we find

$$
\left(\partial_{t}+v \cdot \nabla_{x}\right) f=\lambda^{-1} \int d v_{2} \int_{S_{+}} d n\left(v-v_{2}\right) \cdot n\left[f\left(x, v^{\prime}\right) f\left(x, v_{2}^{\prime}\right)-f(x, v) f\left(x, v_{2}\right)\right] .
$$

The parameter $\lambda$ represents, roughly, the typical length a particle can cover without undergoing any collision (mean free path). (In (10), we just chose $\lambda=1$.)

Remark. After having taken the limit $N \rightarrow \infty$ and $\varepsilon \rightarrow 0$, there is no way to distinguish between incoming and outgoing pair velocities because no trace of the parameter $\varepsilon$ is left in (23) and $n$ plays the role of a random parameter. However, keeping in mind the way we derived the Boltzmann equation, we shall conventionally maintain the name "incoming" for velocities satisfying the condition $\left(v-v_{2}\right) \cdot n \geq 0$ and consequently the pair $v^{\prime}$ and $v_{2}^{\prime}$ is outgoing in (23).

Equation (23) (or equivalently (10)-(11)) is the Boltzmann equation for hard spheres. Such an equation has a statistical nature, and it is not equivalent to the Hamiltonian dynamics from which it has been derived. Indeed the $H$ theorem shows that it is not reversible in time in contrast with the laws of mechanics. We note, incidentally, that this is not the case for the Vlasov equation, which inherits all the properties of the Hamiltonian systems. 
By the analysis on the order of magnitude of the quantities in the game, we deduced that the Boltzmann equation works in special situations only. The condition $N \varepsilon^{2}=O(1)$ means that we consider a rarefied gas, with almost vanishing volume density. After Boltzmann established the equation, Harold Grad [1949; 1958] postulated its validity in the limit $N \rightarrow \infty$ and $\varepsilon \rightarrow 0$ with $N \varepsilon^{2} \rightarrow$ const. as discussed above (this is often called, indeed, the Boltzmann-Grad limit).

There is no contradiction in the irreversibility or in the trend to equilibrium obtained after the limit, when they are strictly speaking false for mechanical systems. However, the arguments above are delicate and require a rigorous, deeper analysis. If the Boltzmann equation is not a purely phenomenological model derived by assumptions ad hoc and justified by its practical relevance, but rather a consequence of a mechanical model, we should derive it rigorously. In particular, the propagation of chaos should not be a hypothesis but the statement of a theorem.

After the formulation of the mathematical problem by Grad, Cercignani [1972] obtained the evolution equation (hierarchy) for the marginals of a hard-sphere system, and this was the starting point to rigorously derive the Boltzmann equation, as accomplished by Lanford [1975] in his famous paper, even though only for a short time interval.

Lanford's theorem is probably the most relevant result regarding the mathematical foundations of the kinetic theory. In fact, it dispelled the many previous doubts on the validity of the Boltzmann equation (although some authors refuse a priori the problem of deriving the equation starting from mechanical systems [Truesdell and Muncaster 1980]).

Unfortunately, the short-time limitation is serious. Only for special systems, as the case of a very rarefied gas expanding in a vacuum, can we obtain a global validity result [Illner and Pulvirenti 1986; 1989]. The possibility of deriving the Boltzmann equation globally in time, at least in cases when we have a good global existence of solutions, is still an open and challenging problem.

We conclude this section with some historical remarks.

Before Boltzmann, Maxwell proposed a kinetic equation that is nothing other than the Boltzmann equation integrated against test functions [Maxwell 1867; 1995]. He considered also more general potentials, in particular inverse-power-law potentials, essentially for the special properties of their cross-sections.

After Lanford's result, the case of smooth short-range potentials has been studied by other authors [King 1975; Gallagher et al. 2014; Pulvirenti et al. 2014], but the validity (or nonvalidity) of the Boltzmann equation in the case of genuine longrange potentials is open.

A rigorous derivation of the hierarchy of equations for hard spheres formally established by Cercignani is obtained in [Spohn 1991; Cercignani et al. 1994; Simonella 2014]. 


\section{The weak-coupling limit and the Landau equation}

The Boltzmann equation is suited to the description of rarefied gases, and one can ask whether a useful kinetic analysis can be applied also to the case of a dense gas. To introduce the problem, let us revisit first the Boltzmann-Grad limit in an alternative way. Let $\varepsilon$ be a small scale parameter denoting the ratio between the microscopic and macroscopic scales, for instance the inverse number of atomic diameters necessary to cover 1 meter or the inverse number of atomic characteristic times necessary to cover 1 second. Then scale space and time by $\varepsilon$ in the equations of motion (in our case, the hard-sphere hierarchy). We need to specify the number of particles $N$. In a box of side 1 , there should be $N \approx \varepsilon^{-3}$ particles if one assumes that the intermolecular distance is of the same order as the molecular diameter. The number of collisions of a given particle per macroscopic unit time would be $\varepsilon^{-1}$. As we have seen, in a low-density regime, $N$ scales differently, namely $N \approx \varepsilon^{-2}$, the number of collisions per unit time is finite and the one-particle distribution function satisfies the Boltzmann equation.

A variety of possible scalings describes different physical situations. For instance, the gas may be dense, $N=O\left(\varepsilon^{-3}\right)$ and the particles weakly interacting via a smooth two-body potential $\varphi$. To express the weakness of the interaction, we assume that $\varphi$ is rescaled by $\sqrt{\varepsilon}$. Since $\varphi$ varies on a scale $\varepsilon$ (in macroscopic units), the force will be $O(1 / \sqrt{\varepsilon})$ and act on a time interval $O(\varepsilon)$. The variation of momentum due to the single scattering is $O(\sqrt{\varepsilon})$, and the number of particles met by a typical particle is $O(1 / \varepsilon)$. Hence, the total momentum variation for unit time is $O(1 / \sqrt{\varepsilon})$. However, in the case of a homogeneous gas and symmetric forces, this variation should be zero in the average. The computation of the variance leads to a result $(1 / \varepsilon) O(\sqrt{\varepsilon})^{2}=O(1)$. Therefore, based on a central-limit type of argument, we expect that in the kinetic limit a diffusion equation in the velocity variable holds. Moreover, even though the force induced by a given particle on a test particle is $O(1 / \sqrt{\varepsilon})$ (i.e., not small as in the mean-field limit), the fact that it produces a small variation on the momentum should be sufficient to ensure propagation of chaos.

At the level of the kinetic equation (that is, assuming propagation of chaos), consider a model of collision with an operator of the form

$$
Q(f, f)=\int d v_{1} \int d p w(p) \delta\left(p^{2}+\left(v-v_{1}\right) \cdot p\right)\left[f^{\prime} f_{1}^{\prime}-f f_{1}\right]
$$

where

$$
f^{\prime}=f(v+p), \quad f_{1}^{\prime}=f\left(v_{1}-p\right) .
$$

Here $p$ is the transferred momentum in the collision, and $w$ is the probability density of having $p$ as an effect of the collision. The $\delta$ expresses energy conservation. 
To express the fact that the transferred momentum is small, let us rescale $w$ as $\left(1 / \varepsilon^{3}\right) w(p / \varepsilon)$ (so that the transferred momenta are $O(\varepsilon)$ ). In addition, let us rescale the inverse mean-free path by a factor $1 / \varepsilon$ to take into account the high density of particles. The collision operator becomes

$$
\begin{aligned}
& Q_{\varepsilon}(f, f)= \frac{1}{\varepsilon^{4}} \int d v_{1} \int d p w\left(\frac{p}{\varepsilon}\right) \delta\left(p^{2}+\left(v-v_{1}\right) \cdot p\right)\left[f^{\prime} f_{1}^{\prime}-f f_{1}\right] \\
&=\frac{1}{2 \pi \varepsilon^{2}} \int d v_{1} \int d p w(p) \int_{-\infty}^{+\infty} d s e^{i s\left(p^{2} \varepsilon+\left(v-v_{1}\right) \cdot p\right)} \\
& \times\left[f(v+\varepsilon p) f\left(v_{1}-\varepsilon p\right)-f(v) f\left(v_{1}\right)\right] \\
&=\frac{1}{2 \pi \varepsilon} \int d v_{1} \int d p w(p) \int_{0}^{1} d \lambda \int_{-\infty}^{+\infty} d s e^{i s\left(p^{2} \varepsilon+\left(v-v_{1}\right) \cdot p\right)} \\
& \times p \cdot\left(\nabla_{v}-\nabla_{v_{1}}\right) f(v+\varepsilon \lambda p) f\left(v_{1}-\varepsilon \lambda p\right) .
\end{aligned}
$$

Here the smooth function $w$, which modulates the collision, is assumed to depend on $p$ through its modulus only. Note that we used a change of variables $p / \varepsilon \rightarrow p$ and the representation formula in $\mathbb{R}^{1}$

$$
\delta(x)=\frac{1}{2 \pi \varepsilon} \int_{-\infty}^{+\infty} d s e^{i s x / \varepsilon} .
$$

To outline the behavior of $Q_{\varepsilon}(f, f)$ in the limit $\varepsilon \rightarrow 0$, we introduce a test function $u$ for which, after a change of variables (here $(\cdot, \cdot)$ denotes the scalar product in $\left.L^{2}(d v)\right)$,

$$
\begin{aligned}
\left(u, Q_{\varepsilon}(f, f)\right)=\frac{1}{2 \pi \varepsilon} & \int d v \int d v_{1} \int d p w(p) \int_{0}^{1} d \lambda \int_{-\infty}^{+\infty} d s \\
& \times e^{i s\left(p^{2}(\varepsilon-2 \varepsilon \lambda)+\left(v-v_{1}\right) \cdot p\right)} u(v-\varepsilon \lambda p) p \cdot\left(\nabla_{v}-\nabla_{v_{1}}\right) f f_{1} \\
=\frac{1}{2 \pi \varepsilon} & \int d v \int d v_{1} \int d p w(p) \int_{0}^{1} d \lambda \int_{-\infty}^{+\infty} d s \\
& \times e^{i s\left(v-v_{1}\right) \cdot p}\left[u(v)-\varepsilon \lambda p \cdot \nabla_{v} u(v)\right] p \cdot\left(\nabla_{v}-\nabla_{v_{1}}\right) f f_{1} \\
+ & \frac{1}{2 \pi} \int d v \int d v_{1} \int d p w(p) \int_{-\infty}^{+\infty} d s e^{i s\left(v-v_{1}\right) \cdot p} u(v) \\
& \times i s p^{2} \int_{0}^{1} d \lambda(1-2 \lambda) p \cdot\left(\nabla_{v}-\nabla_{v_{1}}\right) f f_{1}+O(\varepsilon) .
\end{aligned}
$$

Note now that the term $O\left(\varepsilon^{-1}\right)$ vanishes because of the symmetry $p \rightarrow-p(w$ is even). The last term also vanishes since the integral in $d \lambda$ is zero. As a result,

$$
\begin{aligned}
&\left(u, Q_{\varepsilon}(f, f)\right)=-\frac{1}{4 \pi} \int d v \int d v_{1} \int d p w(p) \int_{-\infty}^{+\infty} d s e^{i s\left(v-v_{1}\right) \cdot p} \\
& \times p \cdot \nabla_{v} u p \cdot\left(\nabla_{v}-\nabla_{v_{1}}\right) f f_{1}+O(\varepsilon) .
\end{aligned}
$$


Therefore, we have recovered (formally) the kinetic equation

$$
\left(\partial_{t}+v \cdot \nabla_{x}\right) f=Q_{L}(f, f)
$$

with a new collision operator

$$
Q_{L}(f, f)=\int d v_{1} \nabla_{v} a\left(\nabla_{v}-\nabla_{v_{1}}\right) f f_{1},
$$

where $a=a\left(v-v_{1}\right)$ denotes the matrix

$$
a_{i, j}(V)=\frac{1}{2} \int d p w(p) \delta(V \cdot p) p_{i} p_{j} .
$$

This matrix can be handled in a better way by introducing polar coordinates:

$$
\begin{aligned}
a_{i, j}(V) & =\frac{1}{2|V|} \int d p|p| w(p) \delta(\hat{V} \cdot \hat{p}) \hat{p}_{i} \hat{p}_{j} \\
& =\frac{B}{|V|} \int d \hat{p} \delta(\hat{V} \cdot \hat{p}) \hat{p}_{i} \hat{p}_{j},
\end{aligned}
$$

where $\hat{V}$ and $\hat{p}$ are the versors of $V$ and $p$, respectively, and

$$
B=\frac{1}{2} \int_{0}^{+\infty} d r r^{3} w(r) .
$$

Note that $B$ is the only parameter describing the interaction appearing in the equation. Finally a straightforward computation yields

$$
a_{i, j}(V)=\frac{B}{|V|}\left(\delta_{i, j}-\hat{V}_{i} \hat{V}_{j}\right) .
$$

The collision operator $Q_{L}$ was introduced by Landau in 1936 [Landau 1965; Lifshitz and Pitaevskiı 1981] for the study of a weakly interacting dense plasma, and (28) is called the Landau equation (sometimes Landau-Fokker-Planck).

The qualitative properties of the solutions to the Landau equation are the same as for the Boltzmann equation regarding the basic conservation laws and the $H$ theorem.

Following the paradigm of kinetic theory, we would like to derive the Landau equation from particle systems. A rigorous proof is however missing, even for short time intervals. We refer to [Boblylev et al. 2013] for a partial result.

\section{Some historical remarks}

The first attempt to implement the program of kinetic theory was due to Boltzmann, who derived his celebrated equation for rarefied gases in 1872 [Boltzmann 1964]. This followed some previous work of Maxwell. He wrote a system of equations 
in 1867 for the moments of the velocity distribution in order to justify the equilibrium measure which inherits his name [Maxwell 1867; 1995].

Boltzmann's work was attacked by several physicists and mathematicians due to the apparent basic contradiction between the $H$ theorem and the reversible nature of Newton's equations. In particular, the Poincaré recurrence theorem seemed to be in contrast with convergence toward an equilibrium state. Boltzmann replied to the criticisms asserting that the equation has a statistical meaning. Of course, he did not have at his disposal the mathematical tools suitable to make this statement more precise. We do not further discuss this interesting aspect and refer the reader to [Cercignani 1998].

In spite of the success of the Boltzmann equation in solving practical problems concerning rarefied gases, the issue of a rigorous justification of the equation remained open for a long time.

As already mentioned, a significant step forward was taken by Grad [1949; 1958], who figured out the scaling limit in which the equation is expected to hold, in the framework of classical mechanics. A second important contribution along the lines of Grad's approach was then made by Cercignani [1972], who established the hierarchy for the hard-sphere system whose first equation was written in Section 3. His analysis was formal, but it opened the way for Lanford's [1975] rigorous result on the short time validity of the Boltzmann equation.

Lanford's result solved the problem of conciliating the Boltzmann equation with the laws of classical mechanics.

On the other hand, even in recent times, the Boltzmann equation has often been considered a useful and successful tool of investigation and not necessarily as a direct consequence of the principles of mechanics. This is, for instance, the position of Truesdell and Muncaster [1980] in their famous monograph:

"We will make no attempt to trace the source of this irreversibility in more general theories or physico-philosophical speculations. Rather, in the spirit of rational mechanics, we shall attempt to determine its specific and rigorous mathematical nature and consequences."

Another attitude was the one of the great probabilist Kac [1956; 1959], who conceived the stochastic dynamics for an $N$-particle system yielding rigorously, in the limit $N \rightarrow \infty$, the homogeneous Boltzmann equation. His work is contemporary with the one of Grad; however, the point of view is very different. A footnote in [Kac 1956] reads,

"This formulation led to the well-known paradoxes which were fully discussed in the classical article of P. and T. Ehrenfest. These writers made it clear 
(a) that the 'Stosszahlansatz' cannot be strictly derivable from purely dynamic considerations and

(b) that the 'Stosszahlansatz' has to be interpreted probabilistically.

The recent attempts of Born and Green, Kirkwood and Bogoliubov to derive Boltzmann's equation from Liouville's equation and hence to justify the 'Stosszahlansatz' dynamically are, in our opinion, incomplete, inasmuch as they do not make it clear at what point statistical assumptions are introduced.

"The 'master equation' approach which we have chosen seems to us to follow closely the intentions of Boltzmann."

The works quoted after point (b) were the first to attempt a justification of kinetic equations based on a hierarchical technique, and the "Stosszahlansatz" is the property of propagation of chaos necessary to close the hierarchy (we refer in particular to [Bogolyubov 1946] for a pioneering analysis including the three classical kinetic equations).

Therefore, Kac's purpose is not just to provide a toy model as it is intended to be strongly related to the physics. A further quotation from the same paper is,

"Since the master equation is truly descriptive of the physical situation, and since existence and uniqueness of the solution of the master equation are almost trivial, the preoccupation with existence and uniqueness theorems for the Boltzmann equation appears to be unjustified on grounds of physical interest and importance."

An important point is that Kac's model is restricted to homogeneous situations (no dependence on positions). Interestingly enough, in the completely different context of numerical simulations of rarefied gases, Bird [1976] constructed the successful scheme known as DSMC (direct-simulation Monte Carlo), which splits the dynamics of a particle system into two parts: free motion and a stochastic interaction closely related to the one of Kac. In other words (without knowing Kac's work), Bird was providing an inhomogeneous stochastic model approximating the Boltzmann equation (see also [Cercignani et al. 1994] and references quoted therein).

Kac was greatly influenced by the famous treatise of Paul and Tatjana Ehrenfest [1959], where the conceptual bases of statistical mechanics are discussed. Here the authors try to explain the nature of the Boltzmann equation and the emergence of irreversibility with the aid of simple examples.

We note, incidentally, that in [Ehrenfest and Ehrenfest 1959] a model is introduced (often called the wind-tree model) in which a light (point) particle collides with a random distribution of square obstacles in the whole plane, in such a way that only four velocities are possible. The set of velocities is $\mathscr{V}=\left\{ \pm e_{1}, \pm e_{2}\right\}$, where 
$e_{i}, i=1,2$, are the versor of the coordinate axes in the plane. An elastic collision of the light particle with an obstacle with sides oriented at $\pi / 4$ is a rotation of the incoming velocity by $\pm \pi / 2$. The corresponding kinetic equation is linear and has the form

$$
\left(\partial_{t}+v \cdot \nabla_{x}\right) f=\frac{1}{2}\left(f\left(v^{\perp}\right)+f\left(-v^{\perp}\right)\right)-f(v)
$$

where $\mathscr{V} \ni v \rightarrow v^{\perp}$ is the rotation of $\pi / 2$.

For the more realistic "Lorentz model" with circular obstacles and velocity set $S^{1}$, a rigorous derivation of the linear Boltzmann equation was obtained by Gallavotti [1999] in a remarkable paper. His approach applies as well to the Ehrenfest wind-tree model.

We also mention a nonlinear version of the wind-tree model, namely the Broadwell model with kinetic equation

$$
\left(\partial_{t}+v \cdot \nabla_{x}\right) f=\left(f\left(v^{\perp}\right) f\left(-v^{\perp}\right)-f(v) f(-v)\right) .
$$

Surprisingly, this equation cannot be derived from the mechanical system of colliding square particles in the plane in the Boltzmann-Grad limit [Uchiyama 1988; Cercignani et al. 1994]. This counterexample shows how delicate a rigorous study of the low-density limit of deterministic systems can be.

As mentioned in Section 4, Landau proposed his kinetic equation for dense gases and plasmas in 1936. He started by assuming the Boltzmann equation with Coulomb cross-section, cutting divergences at short and at long distances. His argument is similar to the one presented here in Section 4, and the problem of the propagation of chaos is pragmatically avoided.

Bogolyubov [1946] works instead with the BBGKY hierarchy and asserts that it would be necessary to obtain the Landau equation starting from particle systems under a suitable scaling limit instead of starting from the Boltzmann equation directly. His discussion amounts to what is nowadays called the "weak-coupling limit" for the Landau equation (see also [Balescu 1975]) and includes an attempt to outline the various regimes in which the kinetic equations are expected to be valid, starting again from the hierarchy.

The Vlasov equation was introduced first in 1938 [Vlasov 1967] to study the time evolution of the distribution function of plasmas consisting of charged particles and long-range forces (for example, Coulomb) in contrast with the Landau equation, which is suited for particles interacting weakly via short-range forces. Actually both equations are needed to retain different aspects of the complicated dynamics of plasmas.

We shall conclude by recalling the famous speech by Hilbert [1902] at the International Congress of Mathematicians in Paris in 1900, where he posed twentythree problems as the basis of mathematical research in the forthcoming century. 
Among these, the sixth is perhaps a less definite problem but rather a broad field of investigation and a prophecy of the modern role of mathematics in physics. It is titled "Mathematical of the Axioms of Physics" and reads,

"The investigations on the foundation of geometry suggest [...] to treat in the same manner, by means of axioms, those physical sciences in which mathematics play an important part; in the first rank are the theory of probabilities and mechanics.

"As to the axioms of the theory of probabilities, it seems to me desirable that their logical investigation should be accompanied by a rigorous and satisfactory development of the method of mean values in mathematical physics, and in particular in the kinetic theory of gases.

"Important investigations by physicists on the foundations of mechanics are at hand [...]. Thus Boltzmann's work on the principles of mechanics suggests the problem of developing mathematically the limiting processes, there merely indicated, which lead from the atomistic view to the laws of the motion of continua."

The necessity of a rigorous approach to the scaling limits starting from fundamental particle models is clearly expressed. Moreover, the role of mathematics in investigating how different mathematical models of reality are connected is outlined:

"[...] Further, the mathematician has the duty to test exactly in each instance whether the new axioms are compatible with the previous ones. The physicist, as his theories develop, often finds himself forced by the results of the experiments to make new hypotheses, while he depends, with respect to the compatibility of the new hypotheses with the old axioms, solely upon these experiments or upon certain physical intuition, a practice which in the rigorously logical building up of a theory is not admissible."

Clarifying the scopes and methodologies of a physicist and a mathematician establishes the role of modern mathematical physics, in which the concept of a mathematical model, as a noncontradictory system of axioms, is fundamental.

The sixth problem of Hilbert can nowadays be further specified; namely there are at least three kinds of convergence which can be analyzed: (1) derive the Boltzmann equation from particle systems, (2) derive the Euler and/or Navier-Stokes equations from the Boltzmann equation and (3) derive the Euler and/or NavierStokes equations from particle systems.

We have remarkable progress regarding points (1) and (2). The first point has been discussed in this note. Regarding point (2), Hilbert [1912] himself introduced 
an expansion which is the basic tool for deriving the Euler equation for compressible fluids, in a suitable scaling limit, starting from the Boltzmann equation. Many rigorous results deriving hydrodynamic laws from the Boltzmann equation have been obtained over recent years. We underline that the hydrodynamic laws are the ones of a perfect gas since we start from a low-density regime. A more challenging problem is the derivation of the Euler equation from particle systems (point (3)). The laws relating density, pressure and temperature are not those of a perfect gas, but they may be computed through the Gibbs state associated with the interacting potential of the system. Such a difficult problem is unsolved on the mathematical side. We mention only the formal computations of the pioneering work by Morrey [1955]. Here the author gives a list of necessary steps to prove that the Euler equation can be obtained from the Newton laws. It is a notable work, having the merit of showing in a logically clear way what the link between the microscopic and macroscopic descriptions of fluids is. See [Esposito and Pulvirenti 2004] for a review of the argument.

\section{Acknowledgment}

We thank Thierry Paul for fruitful discussions.

\section{References}

[Balescu 1975] R. Balescu, Equilibrium and nonequilibrium statistical mechanics, Wiley-Interscience, New York, 1975.

[Bird 1976] G. A. Bird, Molecular gas dynamics, Clarendon, Oxford, 1976.

[Boblylev et al. 2013] A. V. Boblylev, M. Pulvirenti, and C. Saffirio, "From particle systems to the Landau equation: a consistency result", Comm. Math. Phys. 319:3 (2013), 683-702.

[Bogolyubov 1946] N. N. Bogolyubov, Problems of a dynamical theory in statistical physics, Gostekhisdat, Moscow, 1946. In Russian; translated in Studies in statistical mechanics, I, edited by J. de Boer and G. E. Uhlenbeck, North-Holland, Amsterdam, 1962.

[Boltzmann 1964] L. Boltzmann, Lectures on gas theory, University of California, Berkeley, CA, 1964.

[Cercignani 1972] C. Cercignani, "On the Boltzmann equation for rigid spheres", Transport Theory Statist. Phys. 2:3 (1972), 211-225.

[Cercignani 1998] C. Cercignani, Ludwig Boltzmann: the man who trusted atoms, Oxford University, 1998.

[Cercignani et al. 1994] C. Cercignani, R. Illner, and M. Pulvirenti, The mathematical theory of dilute gases, Applied Mathematical Sciences 106, Springer, New York, 1994.

[Dobrušin 1979] R. L. Dobrušin, "Vlasov equations", Funktsional. Anal. i Prilozhen. 13:2 (1979), 48-58. In Russian; translated in Funct. Anal. Appl. 13:2 (1979), 115-123.

[Ehrenfest and Ehrenfest 1959] P. Ehrenfest and T. Ehrenfest, The conceptual foundations of the statistical approach in mechanics, Cornell University, Ithaca, NY, 1959. 
[Esposito and Pulvirenti 2004] R. Esposito and M. Pulvirenti, "From particles to fluids", pp. 1-82 in Handbook of mathematical fluid dynamics, vol. III, edited by S. Friedlander and D. Serre, NorthHolland, Amsterdam, 2004.

[Gallagher et al. 2014] I. Gallagher, L. Saint-Raymond, and B. Texier, From Newton to Boltzmann: hard spheres and short-range potentials, Zurich Lectures in Advanced Mathematics 18, European Mathematical Society, Zürich, 2014.

[Gallavotti 1999] G. Gallavotti, “Grad-Boltzmann limit and Lorentz's gas”, Appendix 1.A2, pp. 48-55 in Statistical mechanics: a short treatise, Springer, Berlin, 1999.

[Grad 1949] H. Grad, "On the kinetic theory of rarefied gases", Comm. Pure Appl. Math. 2:4 (1949), 331-407.

[Grad 1958] H. Grad, "Principles of the kinetic theory of gases", pp. 205-294 in Handbuch der Physik, Band XII: Thermodynamik der Gase, edited by S. Flügge, Springer, Berlin, 1958.

[Hilbert 1902] D. Hilbert, "Mathematical problems", Bull. Amer. Math. Soc. 8:10 (1902), 437-479.

[Hilbert 1912] D. Hilbert, "Begründung der kinetischen Gastheorie", Math. Ann. 72:4 (1912), 562577.

[Illner and Pulvirenti 1986] R. Illner and M. Pulvirenti, "Global validity of the Boltzmann equation for a two-dimensional rare gas in vacuum”, Comm. Math. Phys. 105:2 (1986), 189-203.

[Illner and Pulvirenti 1989] R. Illner and M. Pulvirenti, "Global validity of the Boltzmann equation for two- and three-dimensional rare gas in vacuum: erratum and improved result", Comm. Math. Phys. 121:1 (1989), 143-146.

[Kac 1956] M. Kac, "Foundations of kinetic theory", pp. 171-197 in Proceedings of the Third Berkeley Symposium on Mathematical Statistics and Probability (Berkeley, CA, 1954-1955), vol. III, edited by J. Neyman, University of California, Berkeley, CA, 1956.

[Kac 1959] M. Kac, Probability and related topics in physical sciences: proceedings of the summer seminar (Boulder, CO, 1957), Lectures in Applied Mathematics 1, Interscience, London, 1959.

[King 1975] F. G. King, BBGKY hierarchy for positive potentials, Ph.D. thesis, University of California, Berkeley, 1975, Available at http://search.proquest.com/docview/302766232.

[Landau 1965] L. D. Landau, "The transport equation in the case of Coulomb interactions", Chapter 24, pp. 163-170 in Collected papers of L. D. Landau, edited by D. ter Haar, Pergamon, Oxford, 1965.

[Lanford 1975] O. E. Lanford, III, "Time evolution of large classical systems", pp. 1-111 in Dynamical systems, theory and applications (Seattle, 1974), edited by J. Moser, Lecture Notes in Physics 38, Springer, Berlin, 1975.

[Lifshitz and Pitaevskiı̌ 1981] E. M. Lifshitz and L. P. Pitaevskiı̆, Course of theoretical physics, vol. 10: Physical kinetics, edited by L. D. Landau and E. M. Lifshitz, Pergamon, Oxford, 1981.

[Maxwell 1867] J. C. Maxwell, "On the dynamical theory of gases", Philos. Trans. Roy. Soc. London 157 (1867), 49-88.

[Maxwell 1995] J. C. Maxwell, The scientific letters and papers of James Clerk Maxwell, vol. II: 1862-1873, edited by P. M. Harman, Cambridge University, 1995.

[Morrey 1955] C. B. Morrey, Jr., "On the derivation of the equations of hydrodynamics from statistical mechanics”, Comm. Pure Appl. Math. 8:2 (1955), 279-326.

[Pulvirenti et al. 2014] M. Pulvirenti, C. Saffirio, and S. Simonella, "On the validity of the Boltzmann equation for short range potentials”, Rev. Math. Phys. 26:2 (2014), 1450001.

[Simonella 2014] S. Simonella, "Evolution of correlation functions in the hard sphere dynamics", $J$. Stat. Phys. 155:6 (2014), 1191-1221. 
[Spohn 1991] H. Spohn, Large scale dynamics of interacting particles, Springer, Berlin, 1991.

[Truesdell and Muncaster 1980] C. Truesdell and R. G. Muncaster, Fundamentals of Maxwell's kinetic theory of a simple monatomic gas: treated as a branch of rational mechanics, Pure and Applied Mathematics 83, Academic, New York, 1980.

[Uchiyama 1988] K. Uchiyama, "On the Boltzmann-Grad limit for the Broadwell model of the Boltzmann equation”, J. Statist. Phys. 52:1-2 (1988), 331-355.

[Vlasov 1967] A. A. Vlasov, "The vibrational properties of an electron gas", Usp. Fiz. Nauk. 93 (1967), 444-470. In Russian; translated in Phys. Usp. 10:6 (1968), 721-733.

Received 28 Jul 2016. Accepted 3 Oct 2016.

MARIO PULVIRENTI: pulviren@mat.uniroma1.it

Dipartimento di Matematica, Università di Roma La Sapienza, Piazzale Aldo Moro 5, I-00185 Rome, Italy

SERGio Simonella: s.simonella@tum.de

Zentrum Mathematik, Technische Universität München, Boltzmannstraße 3, D-85748 Garching, Germany 
EDITORIAL BOARD

ANTONIO CARCATERRA

ERIC A. CARLEN

FRANCESCO DELL'ISOLA

RAFFAELE ESPOSITO

ALBERT FANNJIANG

Gilles A. FranCFORT

Pierangelo MARCATI

JEAN-JACQUES MARIGO

PETER A. MARKOWICH

MARTIN OSTOJA-STARZEWSKI

PIERRE SEPPECHER

DAVID J. STEIGMANN

PAUl STEINMANN

PierRe M. SuQueT

MANAGING EDITORS

MICOL AMAR

CORRADO LATTANZIO

ANGELA MADEO

MARTIN OSTOJA-STARZEWSKI

ADVISORY BOARD

ADNAN AKAY

Holm AltenBaCH

MICOL AMAR

HARM ASKES

TEODOR ATANACKOVIĆ

VICTOR BERDICHEVSKY

GUY BOUCHITTÉ

ANDREA BRAIDES

ROBERTO CAMASSA

MAURO CARFORE

ERIC DARVE

FELIX DARVE

ANNA DE MASI

GianPiEtro DEL Piero

EMMANUELE Di BENEDETTO

BERNOLD FIEDLER

IRENE M. GAMBA

DAVID Y. GAO

SERGEY GAVRILYUK

TIMOTHY J. HEALEY

DOMINIQUE JEULIN

ROGER E. KHAYAT

CORRADO LATTANZIO

ROBERT P. LIPTON

ANGELO LUONGO

ANGELA MADEO

JUAN J. MANFREDI

CARLO MARCHIORO

GÉRARD A. MAUGIN

ROBERTO NATALINI PATRIZIO NEFF

ANDREY PIATNITSKI

ERRICO PRESUTTI

MARIO PULVIRENTI

LUCIO RUSSO

Miguel A. F. SANJUAN

PATRICK SElVADURAI

ALEXANDER P. SEYRANIAN

MIROSLAV ŠILHAVÝ

GUIDO SWEERS

ANTOINETTE TORDESILLAS

LEV TRUSKINOVSKY

JUAN J. L. VELÁZQUEZ VINCENZO VESPRI ANGELO VULPIANI msp.org/memocs

Università di Roma "La Sapienza", Italia

Rutgers University, USA

(CO-CHAIR) Università di Roma "La Sapienza", Italia

(TREASURER) Università dell'Aquila, Italia

University of California at Davis, USA

(CO-CHAIR) Université Paris-Nord, France

Università dell'Aquila, Italy

École Polytechnique, France

DAMTP Cambridge, UK, and University of Vienna, Austria

(CHAIR MANAGING EDITOR) Univ. of Illinois at Urbana-Champaign, USA

Université du Sud Toulon-Var, France

University of California at Berkeley, USA

Universität Erlangen-Nürnberg, Germany

LMA CNRS Marseille, France

Università di Roma "La Sapienza", Italia

Università dell'Aquila, Italy

Université de Lyon-INSA (Institut National des Sciences Appliquées), France

(CHAIR MANAGING EDITOR) Univ. of Illinois at Urbana-Champaign, USA

Carnegie Mellon University, USA, and Bilkent University, Turkey

Otto-von-Guericke-Universität Magdeburg, Germany

Università di Roma "La Sapienza", Italia

University of Sheffield, UK

University of Novi Sad, Serbia

Wayne State University, USA

Université du Sud Toulon-Var, France

Università di Roma Tor Vergata, Italia

University of North Carolina at Chapel Hill, USA

Università di Pavia, Italia

Stanford University, USA

Institut Polytechnique de Grenoble, France

Università dell'Aquila, Italia

Università di Ferrara and International Research Center MEMOCS, Italia

Vanderbilt University, USA

Freie Universität Berlin, Germany

University of Texas at Austin, USA

Federation University and Australian National University, Australia

Université Aix-Marseille, France

Cornell University, USA

École des Mines, France

University of Western Ontario, Canada

Università dell' Aquila, Italy

Louisiana State University, USA

Università dell'Aquila, Italia

Université de Lyon-INSA (Institut National des Sciences Appliquées), France University of Pittsburgh, USA

Università di Roma "La Sapienza”, Italia

Université Paris VI, France

Istituto per le Applicazioni del Calcolo "M. Picone", Italy

Universität Duisburg-Essen, Germany

Narvik University College, Norway, Russia

Università di Roma Tor Vergata, Italy

Università di Roma "La Sapienza”, Italia

Università di Roma “Tor Vergata”, Italia

Universidad Rey Juan Carlos, Madrid, Spain

McGill University, Canada

Moscow State Lomonosov University, Russia

Academy of Sciences of the Czech Republic

Universität zu Köln, Germany

University of Melbourne, Australia

École Polytechnique, France

Bonn University, Germany

Università di Firenze, Italia

Università di Roma La Sapienza, Italia

MEMOCS (ISSN 2325-3444 electronic, 2326-7186 printed) is a journal of the International Research Center for the Mathematics and Mechanics of Complex Systems at the Università dell'Aquila, Italy.

Cover image: "Tangle” by $\odot$ John Horigan; produced using the Context Free program (contextfreeart.org).

PUBLISHED BY

7 mathematical sciences publishers

nonprofit scientific publishing

http://msp.org/

(C) 2016 Mathematical Sciences Publishers 
Special issue in honor of

Lucio Russo

Lucio Russo: A multifaceted life

Raffaele Esposito and Francesco dell'Isola

The work of Lucio Russo on percolation Geoffrey R. Grimmett

"Mathematics" and "physics" in the science of harmonics Stefano Isola

From quantum to classical world: emergence of trajectories in a quantum system Rodolfo Figari and Alessandro Teta

Propagation of chaos and effective equations in kinetic theory: a brief survey Mario Pulvirenti and Sergio Simonella

What decides the direction of a current? Christian Maes

A remark on eigenvalue perturbation theory at vanishing isolation distance Fiorella Barone and Sandro Graffi

Some results on the asymptotic behavior of finite connection probabilities in percolation Massimo Campanino and Michele Gianfelice

Correlation inequalities for the Potts model Geoffrey R. Grimmett

Quantum mechanics: some basic techniques for some basic models, I: The models Vincenzo Grecchi

Quantum mechanics: some basic techniques for some basic models, II: The techniques Vincenzo Grecchi

On stochastic distributions and currents

Vincenzo Capasso and Franco Flandoli

A note on Gibbs and Markov random fields with constraints and their moments Alberto Gandolfi and Pietro Lenarda

Quantum mechanics: light and shadows (ontological problems and epistemic solutions) Gianfausto Dell'Antonio

MEMOCS is a journal of the International Research Center for the Mathematics and Mechanics of Complex Systems at the Università dell' Aquila, Italy.

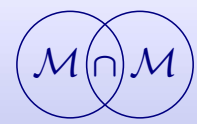

